J. Biosci., Vol. 7, Numbers 3 \& 4, June 1985, pp. 345-357. (C) Printed in India.

\title{
A quantitative analysis of germ cells and the histone variants in the testes of vitamin A-deficient rats and during subsequent repletion with vitamin $\mathrm{A}$
}

\author{
EMMANUAL UNNI and M. R. S. RAO* \\ Department of Biochemistry, Indian Institute of Science, Bangalore 560012, India \\ MS received 13 November 1984; revised 12 April 1985
}

\begin{abstract}
A quantitative analysis of the different types of germ cells present in the seminiferous tubules of vitamin A-deficient-retinoate maintained rats revealed that the number of pachytene spermatocytes and spermatogonia was greatly reduced in the deficient rats. Spermatids were virtually absent in the deficient tubules which contained mostly spermatogonia and preleptotene spermatocytes along with the Sertoli cells. There was no change in the number of Sertoli cells present in the tubules of deficient rats as compared to that of normal rats. Following supplementation of retinyl acetate to vitamin A-deficient-retinoate maintained rats, there was an immediate thinning of the germinal epithelium resulting from the sloughing off of the damaged spermatocytes which were beyond repair. However, after 12 days of vitamin A supplementation fresh batch of pachytene spermatocytes started appearing while by day 16 round spermatids could be seen.

Analysis of the acid soluble proteins from nuclei on different types of Polyacrylamide gel electrophoretic systems has revealed that the levels of the testis specific histone variants Hlt, TH2A and TH2B, synthesized predominantly in the pachytene spermatocytes were greatly reduced in the testes of retinoate maintained rats. Following supplementation of retinyl acetate for either 4 days or 8 days the levels of these histone variants further decreased which correlated with the decrease in the number of pachytene spermatocytes. However, by day 12 of supplementation onwards, their levels started increasing and reached near normal levels by day 24 of vitamin A-supplementation.
\end{abstract}

Keywords. Spermatogenesis; germ cells; histone variants; vitamin A deficiency and repletion.

\section{Introduction}

Spermatogenesis in the mammalian testis is accompanied by a sequential transition of the basic proteins of the chromatin (Kumaroo et al., 1975; Meistrich et al., 1978). There have been extensive studies on the appearance of new histone variants at specific stages of germ cell development in the rat testis. For example, testis specific histones Hlt and TH2B start appearing in pachytene spermatocytes while the histones X2 and Hla, which are not truly testis-specific, are present already in high levels in spermatogonia (Seyedin and Kistler, 1980; Meistrich et al., 1978; Brock et al., 1980). In a recent study Trostle-Weige et al. (1983) have shown that rat testis also contains a variant of histone $\mathrm{H} 2 \mathrm{~A}$, namely $\mathrm{TH} 2 \mathrm{~A}$, which appears in pachytene spermatocytes. The various stages at which histone $\mathrm{H} 1$ subspecies are synthesized have been well documented by the elegant

* To whom correspondence should be addressed.

Abbreviations used: SDS, Sodium dodecyl sulphate; PAGE, Polyacrylamide gel electrophoresis. 
studies of Bucci et al., (1982) using pure populations of germ cells. During stages 12-15 of spermiogenesis, another group of testis-specific proteins termed TP, TP2 and TP4 replace all the somatic and testis-specific histone variants, which ultimately are replaced by an arginine-rich protein S1, the major basic protein present in the mature spermatozoan (Meistrich et al., 1978).

The process of spermatogenesis has been shown to be greatly impaired in vitamin A-deficient rats (Mason, 1933; Howell et al., 1963). Earlier histological and more recent ultrastructural studies have shown that in the testis of vitamin A deficient rats, (i) there is a sloughing off of the germ cells from the epithelium (Mason, 1933; Howell et al. 1965), (ii) an arrest of spermatogenesis at the preleptotene stage (Huang and Hembree, 1979) and (iii) a decrease in the mitotic activity of spermatogonia (Unni et al., 1983). It has further been shown that one of the primary sites of action of vitamin A in rat testis is the Sertoli cell (Unni et al., 1983).

Since the testis-specific histone variants are synthesized at definite stages of germ cell differentiation, they serve as reliable biochemical markers in evaluating the steps of germ cell differentiation that are affected by vitamin A-deficiency. We had shown earlier that the testis-specific protein TP was absent in the testes of vitamin A-deficient rats (Rao et al., 1980). In this communication, we report a more detailed study of the effect of vitamin A deficiency on the testis specific histone variants. We have also made a quantitative analysis of the types of cells present in the seminiferous tubules of such rats.

\section{Materials and methods}

Male albino weanling (21 day old) rats of this Institute strain, originally derived from Wistar strain, were grouped into five batches, namely A, B, C, D and E. All rats were maintained on a vitamin A-deficient diet prepared according to Malathi et al., (1963). Rats belonging to batch A were supplemented with $280 \mu \mathrm{g} / \mathrm{rat} /$ week of retinyl acetate from the beginning and represented controls. The animals in the other four batches were allowed to grow on vitamin A-deficient diet till they attained the weight plateau stage corresponding to a state of mild vitamin A deficiency (60 days, batch B). The rats in batch $\mathrm{C}$ were kept exclusively on the deficient diet till they started losing weight and represented the acutely deficient rats (70 days). The remaining two batches of animals were given oral supplements of retinoic acid as the disodium salt at a dose of $40 \mu \mathrm{g} / \mathrm{rat} /$ day for 30 consecutive days (90-95 days). At the end of such supplementation, one batch representing retinoate maintained rats was designated as 'batch D'. Batch E received $100 \mu \mathrm{g}$ retinyl acetate per animal on alternate days. Representative animals were sacrificed on days $4,8,12,16$ and 24 following this last treatment (replenished group). It has been shown that while supplementation of retinoic acid to vitamin A-deficient rats supports their growth, it does not replace vitamin A alcohol in spermatogenesis (Howell et al., 1963). Thus, retinoate maintained rats can be considered equivalent to vitamin A-deficient rats with respect to spermatogenesis.

All rats were sacrificed by cervical dislocation at $11.00 \mathrm{~A}$. M. on the given day and a small portion of the testis was removed and fixed in Bouin's fluid. Two min later they were cut transversely into 3-4 $\mathrm{mm}$ pieces and kept in the fixative for $8 \mathrm{~h}$. Paraffin blocks 
were made after washing and dehydration. Sections of $34 \mu$ thickness were stained either with Harris hematoxylin and eosin or by periodic acid-Schiff method and counter stained with Harris hematoxylin and examined under a Zeiss photomicroscope III. For counting the types of cells present in cross sections of tubules, 100 tubules per animal were randomly taken and the data are presented as the average \pm S.D. of three such observations. The number of cells given is only a gross number and not corrected using the Abercrombie's formula and Sertoli cell correction factor. The remaining portions of the testes were processed for the isolation of nuclei and analysis of the nuclear acid-soluble proteins (Rao et al., 1980). Electrophoretic analysis of the acid soluble proteins on $15 \%$ Polyacrylamide acid-urea gel was done according to the procedure described by Panyim and Chalkley (1969), while the analysis of H1histones was done on sodium dodecyl sulphate (SDS)-15\% Polyacrylamide gel (PAGE) (Laemmli, 1970) and also by the two dimensional system of Seyedin and Kistler (1980) with acid-urea gel in the first dimension and SDS gel in the second. Analysis of the total histones on acid-urea-Triton X-100 gel was carried out according to the method of Alfageme et al., (1974). For quantitating the individual histone H1 subtypes which were separated on the two dimensional system of Seyedin and Kistler (1980), the protein bound Coomassie blue was eluted from the various spots with $60 \%$ formic acid according to the method of Gibson and Gracy (1979) and the absorbance was measured at $600 \mathrm{~nm}$. The recovery of Coomassie blue stain in this elution process was $90 \%$.

\section{Results and discussion}

\section{Growth curve}

When weanling rats were placed on a vitamin A-free diet, they grew like normal control animals till the vitamin A reserve in the liver was depleted. They attained weights around $95 \mathrm{~g}$ by that time (figure 1). The weights of the animals on the deficient diet remained constant for 6-7 days, when they were 60-63 days old. After this the weight decreased until death, which occurred 7-9 days later. When retinoic acid was fed to vitamin A-deficient rats there was an increase in body weight of 5-6 g/day which continued steadily till the animals were sacrificed 30 days later. No mortality was noticed when rats were maintained on retinoic acid following mild vitamin A deficiency.

\section{Quantitative aspects of germ cells and Sertoli cells}

There are number of reports on the types of cells present in the testes of rats subjected to mild and acute vitamin A deficiency. Recently, Mitranond et al., (1979) examined the kinetics of germ cell depletion during vitamin A deficiency. With the onset of vitamin A deficiency, there was a gradual decrease in the number of spermatids and spermatocytes. In the acute stage, the seminiferous tubules were left with a small population of spermatogonia amidst Sertoli cells. We have confirmed these observations (data not shown) and are presenting here the kinetics of repletion of germ cells once vitamin A supplement is offered to retinoate maintained rats.

Figure 2 illustrates the types of cells present in the tubules of normal rats (A), retinoate maintained rats $(B)$ and those of the replenished group $(\mathrm{C}-\mathrm{H})$. The average 


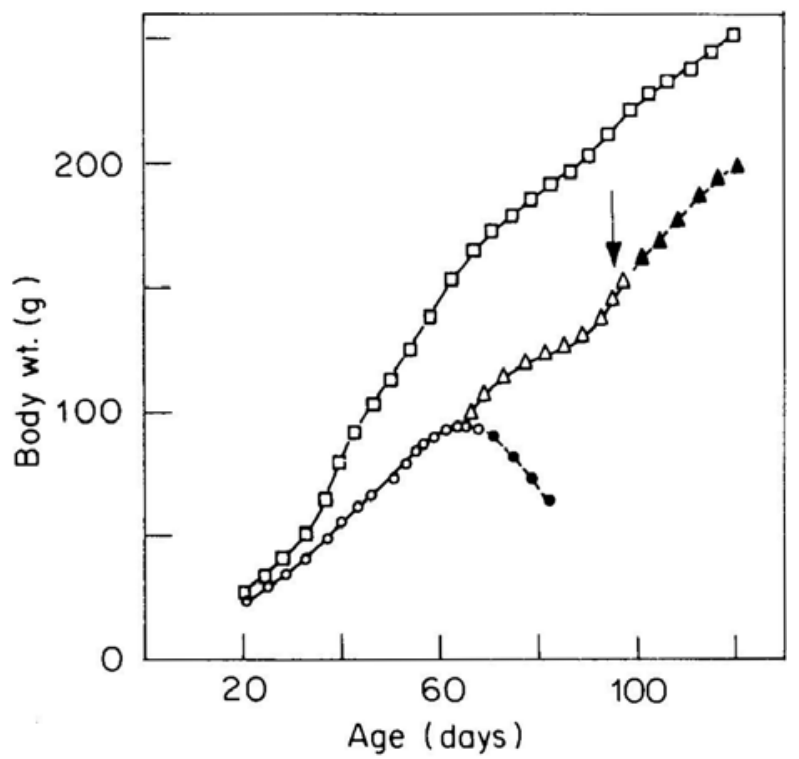

Figure 1. Growth curves of normal, vitamin A-deficient, retinoic acid maintained rats and also of those supplemented with retinyl acetate. ( $\square)$, Normal; (०), mildly vitamin A-deficient; $(\bullet)$, acutely vitamin A-deficient; $(\Delta)$,retinoic acid maintained; $(\boldsymbol{\Delta})$, retinyl acetate supplemented after being on retinoic acid. The arrow indicates the time at which retinyl acetate supplementation was started.

number of different types of cells per cross section of a seminiferous tubule is assembled in figure 3. It can be seen from figure $2 \mathrm{~B}$ that the testes of retinoate maintained rats contained mainly spermatogonia and primary spermatocytes along with the Sertoli cells. However, the populations of spermatogonia and primary spermatocytes were reduced by $77 \%$ and $80 \%$ respectively as compared to the controls. There was a marginal increase in the number of Sertoli cells per cross section of a tubule as a result of a decrease in the tubule diameter from $209 \mu$ in normal to $114 \mu$ in retinoate maintained rats as a consequence of reduction in germ cells. Many of the spermatocytes were in a degenerate state in the retinoate maintained rats (figure 2B). On supplementation with vitamin A they were sloughed of resulting in the thinning of the germinal epithelium (figure 2C). A similar sloughing of of the germ cells following vitamin A was also noticed in our earlier study where we had shown that these sloughed off cells accumulate in the epididymis (Unni et al., 1983).

Active mitosis was noticed in the spermatogonial compartment of testes of retinoate maintained rats following 4 days of supplementation with vitamin A (figure 2C). This was reflected in an increase in the number of spermatogonia per cross section of the tubule. By the 8th day of supplementation their number had reached near normal levels (figures 2D and 3). One can also find a limited number of pachytene spermatocytes in figure 2D. By the 12th day after supplementation, the tubules contained a greater number of pachytene spermatocytes (figure 2E) and by day 24, most of the tubules contained an appreciable number of pachytene spermatocytes (figure $2 \mathrm{H}$ ) reaching $74 \%$ of controls (figure 3). In about $8 \%$ of the tubules in the testes of 16 th day 


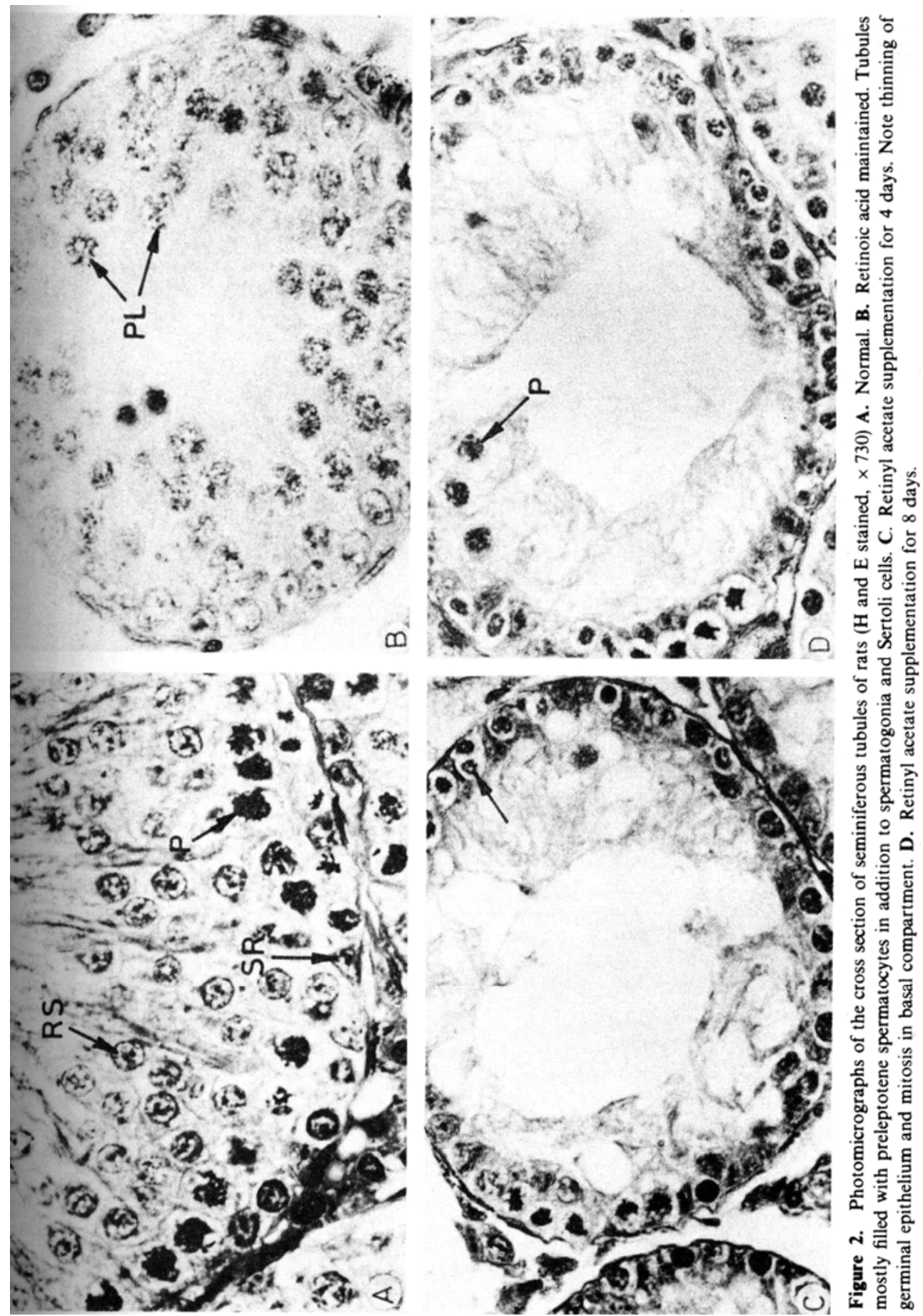



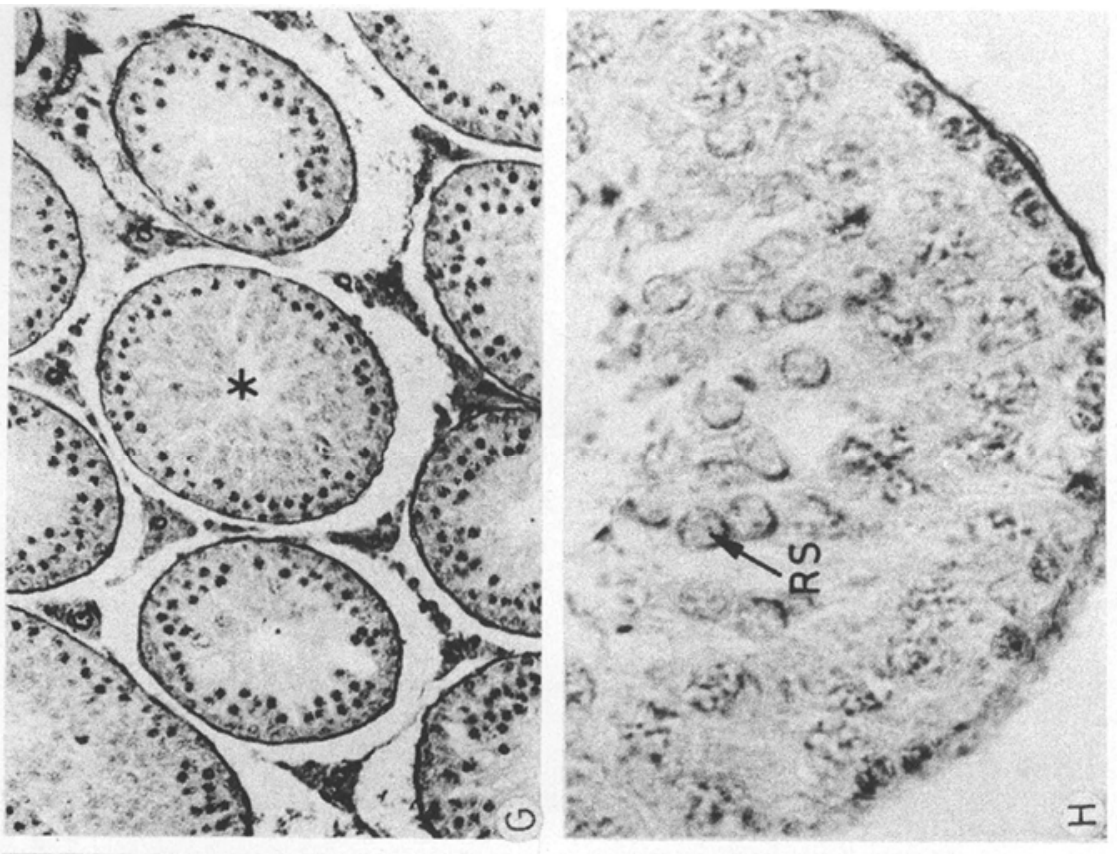

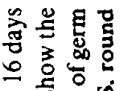

옹. 용

들 氙

要官总

드을

产

卷

政

造专它

숭

讨苦

究造 흥

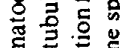

迢密

苍昆

窟高

苔.

这焉

造 壳
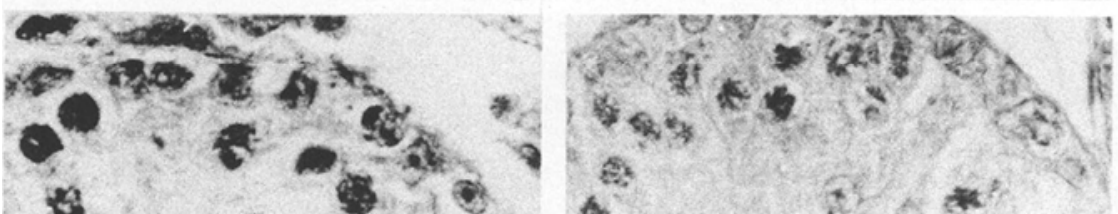

苋氮

言

至

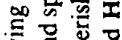

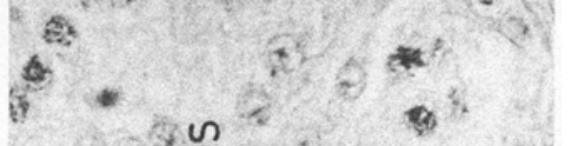

일

은

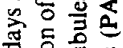
अं 5
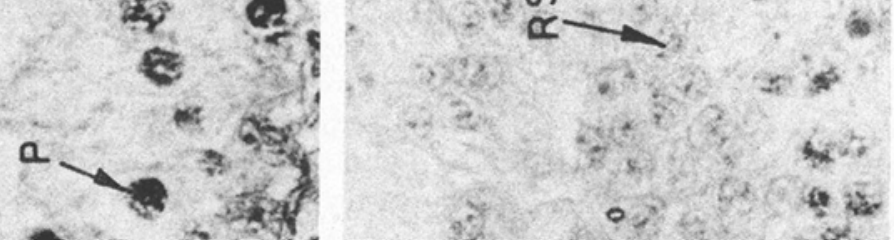

폴

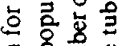

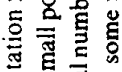

昰范要.

综

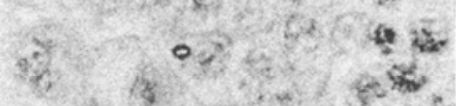

帘

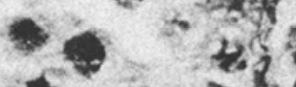

菏

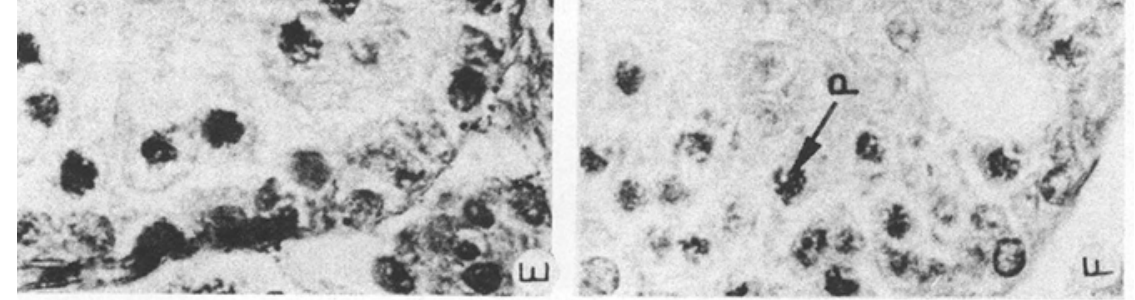

总总

就若

$\propto$

बi

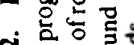

i

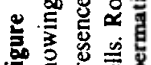




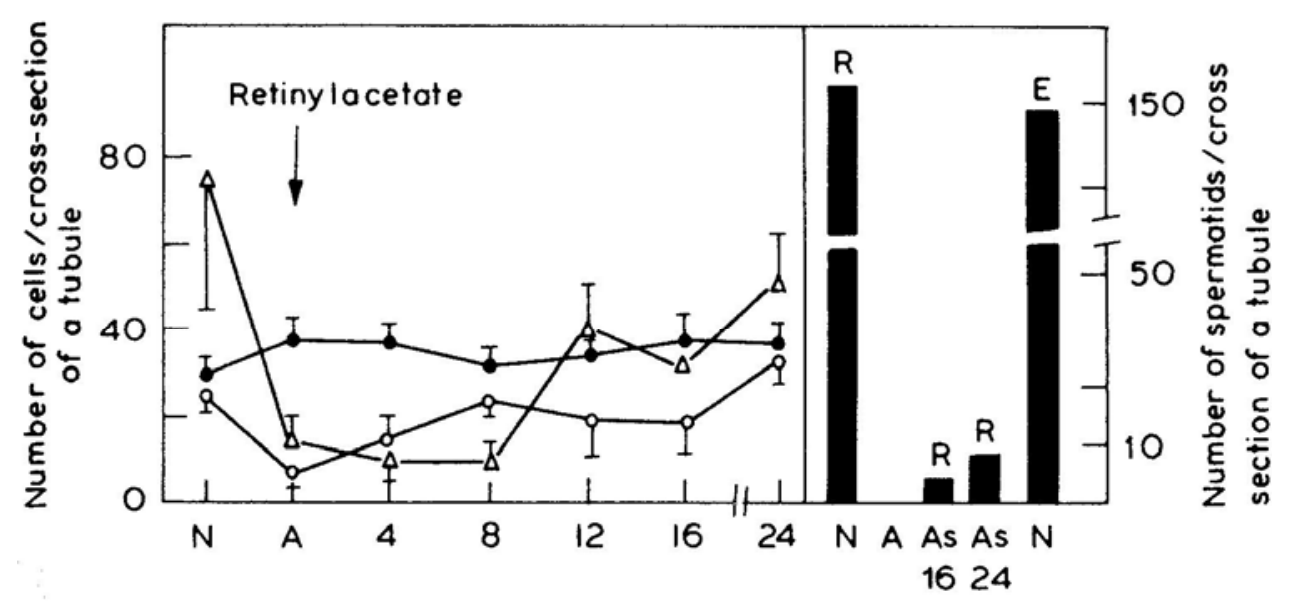

Treatment and days of supplementation

Figure 3. Kinetics of regeneration of different cell types following supplementation of retinyl acetate to retinoate maintained rats. Each value is an average \pm S.D. of three independent observations made in a total of 100 tubules in each observation. N, Normal; A, retinoic acid maintained; AS, retinyl acetate supplemented; R, round spermatids; E, elongating spermatids; $(\bullet)$, Sertoli cells; $(\circ)$, spermatogonia; $(\Delta)$, primary spermatocytes.

(figure $2 \mathrm{~F}$ ) and 24th day (figure $2 \mathrm{H}$ ) after supplementation, round spermatids were also noticed. It is presumed that they are derived from the preleptotene spermatocytes that persisted in the testes of retinoate maintained rats and are not the product of a fresh wave of spermatogenesis, since it takes 30 days for Al spermatogonium to give rise to round spermatids in the testes of normal rats (Courot et al., 1970; Clermont, 1972). This implies that in vitamin A-deficient-retinoate maintained rats a small percentage of healthy preleptotene spermatocytes survive and on supplementation of the diet with vitamin A they progress through the remaining stages of spermatogenesis.

\section{Analysis of somatic and testis-specific histone variants}

The electrophoretic pattern of total acid-soluble nuclear proteins obtained from the testes of rats of different vitamin A status on an acid-urea $15 \%$ PAGE is shown in figure 4. As can be seen from the figure, the levels of THl (Hla $+\mathrm{Hlt})$ and X2 were lower in mild vitamin A-deficient (lane B) and in retinoate maintained rats (lane D) as compared to those in normal rats (lane A). They were significantly reduced in acutely vitamin A-deficient rats (lane $C$ ). On supplementation of retinyl acetate to the retinoate maintained rats for 16 days (lane E), there was a considerable recovery in histone THI and X2 levels. Histone TH1 which behaves as a single band in an acid-urea gel is composed of two histone H1 subtypes, namely Hla and Hlt. In the rat testes, there are 7 histone H1 subtypes and they can be separated into 5 bands on one dimensional SDSPAGE as shown in figure 5. The uppermost band is comprised of 3 histone subtypes $\mathrm{Hlb}$, d and e followed by Hla, Hlc, $\mathrm{Hl}^{\circ}$ and Hlt (Seyedin and Kistler, 1980). It is clear from lanes $\mathrm{A}$ and $\mathrm{F}$ in figure 5 that histone $\mathrm{H} 1 \mathrm{t}$ is specific to testes as it is not present in the acid-soluble nuclear proteins obtained from spleen. Comparison of the acid-soluble 


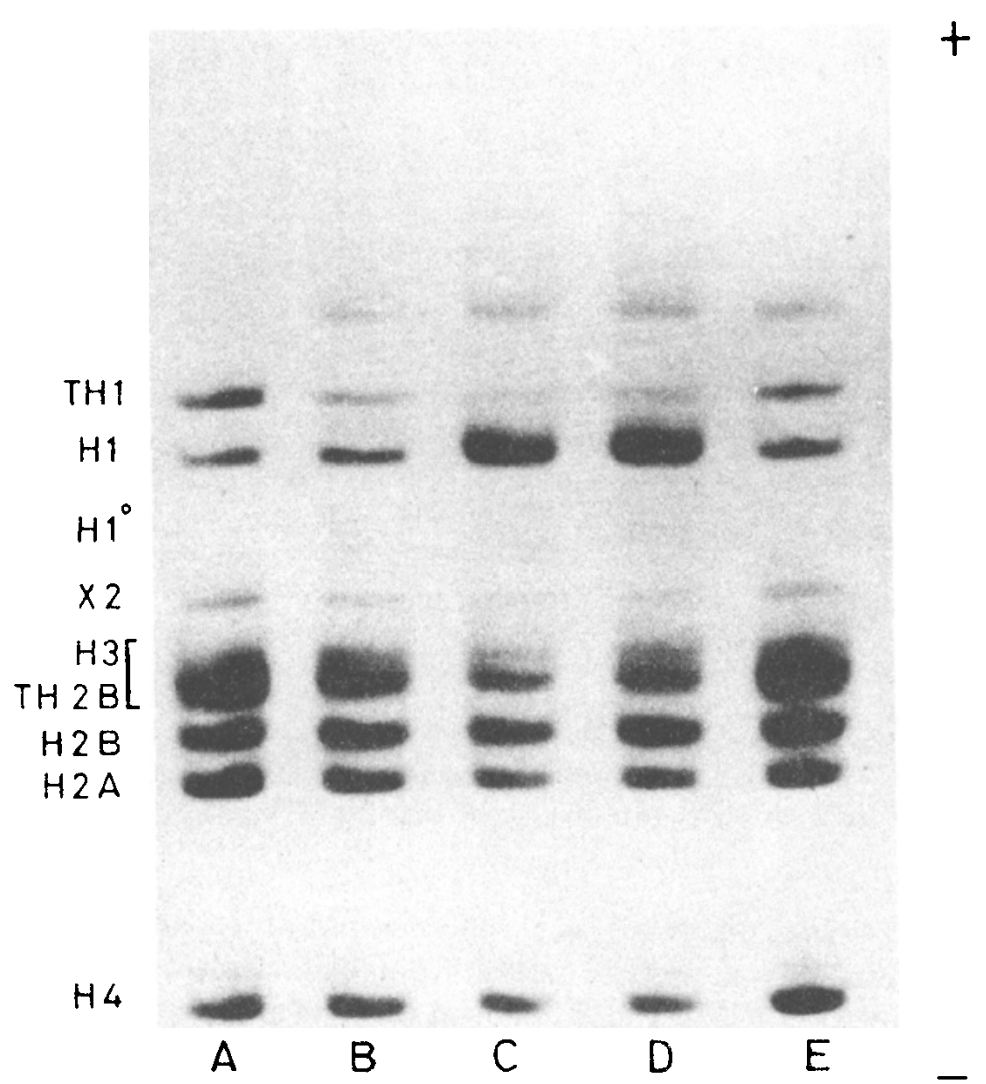

Figure 4. Electrophoretic analysis of acid-soluble nuclear proteins of nuclei from the testes of different vitamin A status on a $15 \%$ Polyacrylamide acid-urea gels. Approximately $100 \mu \mathrm{g}$ of acid soluble proteins were analysed on the acid-urea gel according to the procedure of Panyim and Chalkley (1969). A, Normal, B, mildly vitamin A-deficient; C, acutely vitamin Adeficient; D, retinoic acid maintained and E, retinoic acid maintained rats supplemented with retinyl acetate for 16 days.

nuclear proteins of testes of mildly vitamin A-deficient (lane C), acutely deficient (lane B) as well as retinoate maintained rats (lane D) with that of normal (lane A) clearly showed that the level of histone $\mathrm{H} 1 \mathrm{t}$ was greatly reduced in the former groups of rats. On the other hand, the level of histone $\mathrm{H}^{0}$ increased significantly in the testes of acutely deficient rats (lane B). Recently Bucci et al. (1982) have shown that histone $\mathrm{H} 1^{0}$ is mainly contributed by the somatic cells of the seminiferous tubules and hence its increased relative levels in the testes of acutely deficient rats probably reflects the relative increase in the somatic cells as a consequence of the loss of germ cells.

Employing the two dimensional electrophoretic system of Seyedin and Kistler (1980), we have separated the single band ofhistone Hlof acid-urea PAGE system into two spots namely Hlbde and H1c and the histone TH1 band into H1a and H1t (figure 6) for purposes of quantitation. The nuclear acid-soluble proteins from the testes of 

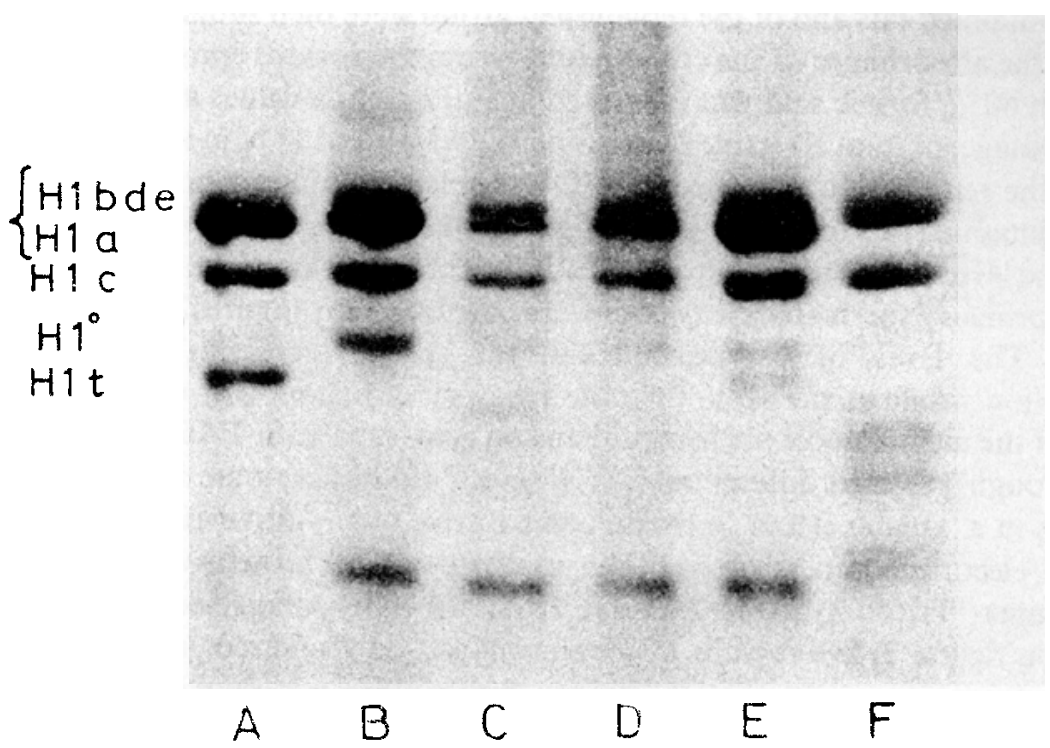

Figure 5. Electrophoretic analysis of acid-soluble nuclear proteins of nuclei from the testes of rats of different vitamin A status on a SDS-15\% PAGE. Approximately $150 \mu \mathrm{g}$ of acid soluble proteins were analysed on the SDS gel according to the method of Laemmli (1970). A, Normal, B, acutely vitamin A-deficient; C, mildly vitamin A-deficient; D, retinoic acid maintained; E, retinoic acid maintained rats supplemented with retinyl acetate for 16 days and $\mathrm{F}$, spleen of normal rat.

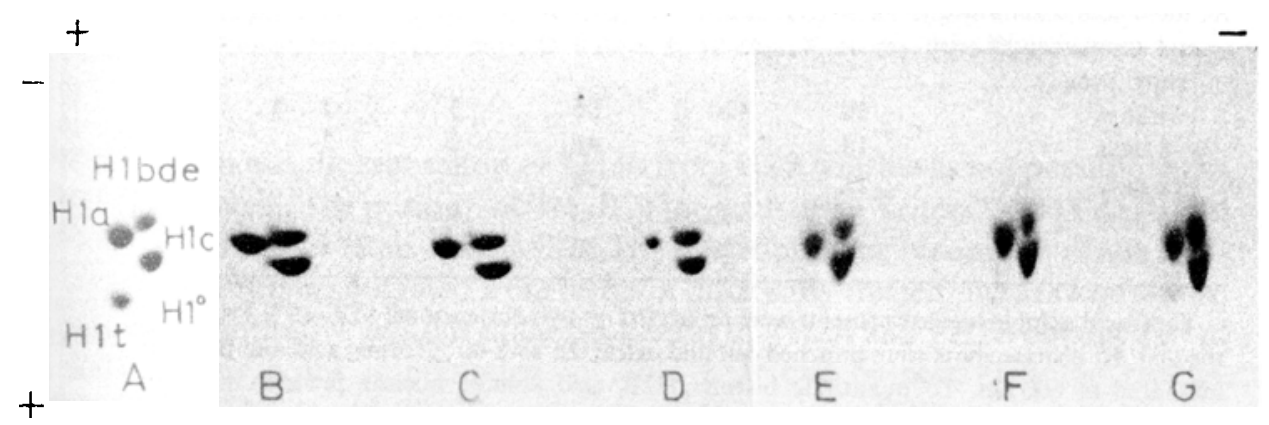

Figure 6. Two dimensional PAGE pattern of $\mathrm{H} 1$ histones. Approximately $150 \mu \mathrm{g}$ of acidsoluble nuclear proteins from the testes of different animals were electrophoresed in the first dimensions on a $15 \%$ Polyacrylamide acid-urea gel and the region corresponding to THl (Hla $+\mathrm{H} 1 \mathrm{t})$ and $\mathrm{H} 1$ region were cut out and electrophoresed in the second dimension on $15 \%$ SDS PAGE. A, Normal; B, retinoic acid maintained; C-G, retinyl acetate supplementation for $\mathrm{C}, 4$ days; D, 8 days; E, 12 days; F, 16 days; and G, 24 days 
retinoate maintained rats and of the replenished group were then subjected to such an analysis and the absorbance of the colour eluted from the various spots of histone H1 subtypes with $60 \%$ formic acid was measured at $600 \mathrm{~nm}$. The values are expressed as relative percentage of total $\mathrm{H} 1$ (table 1). Histone Hlt which was $19 \%$ in normal rats was only $4 \%$ in the retinoate maintained rats. On supplementation of retinyl acetate to retinoate maintained rats for either 4 days (figure 6C) or 8 days (figure 6D), there was a decrease in the levels of histones Hlt as well as Hla (table 1). This decrease is due to the depletion of primary spermatocytes which were beyond repair (figures $2 \mathrm{C}$ and 3 ; Unni et al., 1983). The levels of histones Hlt and Hla started increasing by day 12 of supplementation while at the same time the recovery of histone Hlt was $59 \%$.

Analysis of the acid-soluble nuclear proteins on acid-urea $15 \%$ PAGE described in figure 4, although revealed differences in THl levels, did not separate the nucleosome core histones in a greater detail so that a comparative quantitative analysis could be made. Hence, electrophoretic analysis of the total acid-soluble proteins was also carried out on acid-urea-Triton X-100 $12 \%$ PAGE as described by Alfageme et al., (1974). As can be seen in figure 7, five regions of separation can be visualized. In the region of histone $\mathrm{H} 2 \mathrm{~A}$, four subtypes of $\mathrm{H} 2 \mathrm{~A}$ including the one corresponding to testis-specific variant TH2A were well separated, while in the region of histone H1, two bands corresponding to THI and $\mathrm{H} 1$ similar to the ones obtained in the acid-urea PAGE were seen. Histone H3 got split into two subtypes H3.1 and H3.2. The important advantage

Table 1. Relative levels of histone $\mathrm{H} 1$ subspecies and histones $\mathrm{TH} 2 \mathrm{~A}$ and $\mathrm{TH} 2 \mathrm{~B}$ in the testes of differently treated rats.

\begin{tabular}{|c|c|c|c|c|c|c|c|}
\hline \multirow[b]{2}{*}{ Treatment } & \multicolumn{7}{|c|}{ Relative percentage } \\
\hline & Hla & Hlbde & Hlc & Hlt & $\mathbf{H} \mathbf{l}^{\circ}$ & TH2A* & TH2B $†$ \\
\hline Normal & 28 & 23 & 29 & 19 & 1 & 25 & 53 \\
\hline $\begin{array}{l}\text { Retinoic acid } \\
\text { maintained }\end{array}$ & 32 & 27 & 34 & 4 & 3 & 18 & 32 \\
\hline $\begin{array}{l}\text { Retinoic acid u } \\
\text { and supplem } \\
\text { retinyl aceta }\end{array}$ & & & & & & & \\
\hline-4 days & 28 & 30 & 36 & 3 & 3 & 8 & 17 \\
\hline-8 days & 16 & 39 & 41 & 2 & 2 & 14 & 19 \\
\hline-12 days & 21 & 33 & 38 & 7 & 1 & 15 & 37 \\
\hline-16 days & 27 & 32 & 32 & 8 & 1 & 18 & 34 \\
\hline-24 days & 31 & 27 & 30 & 11 & 1 & 22 & 43 \\
\hline
\end{tabular}

Total acid soluble nuclear proteins were separated on two dimensional SDS-15\% PAGE as mentioned in the text. H1 histone spots were punched out and extracted with $60 \%$ formic acid and the colour eluted was measured at $600 \mathrm{~nm}$. To quantitate histone $\mathrm{H} 2 \mathrm{~B}$, acid soluble nuclear proteins were separated on a Triton-acid-urea 12\% PAGE. Individual lanes were then scanned in a Beckman Model 26 double beam spectrophotometer at $620 \mathrm{~nm}$. The relative percentages of $\mathrm{H} 1$ subspecies are from figure 6 and are with respect to total $\mathrm{H} 1$ subspecies.

* The relative percentage of TH2A is from figure 7 and is with respect to total H2A's (X2, TH2A, H2A.1, H2A.2) which is taken as $100 \%$.

${ }^{\dagger}$ The relative percentage of TH2B is from figure 7 and is with respect to H2B and TH2B which is taken together as $100 \%$. 


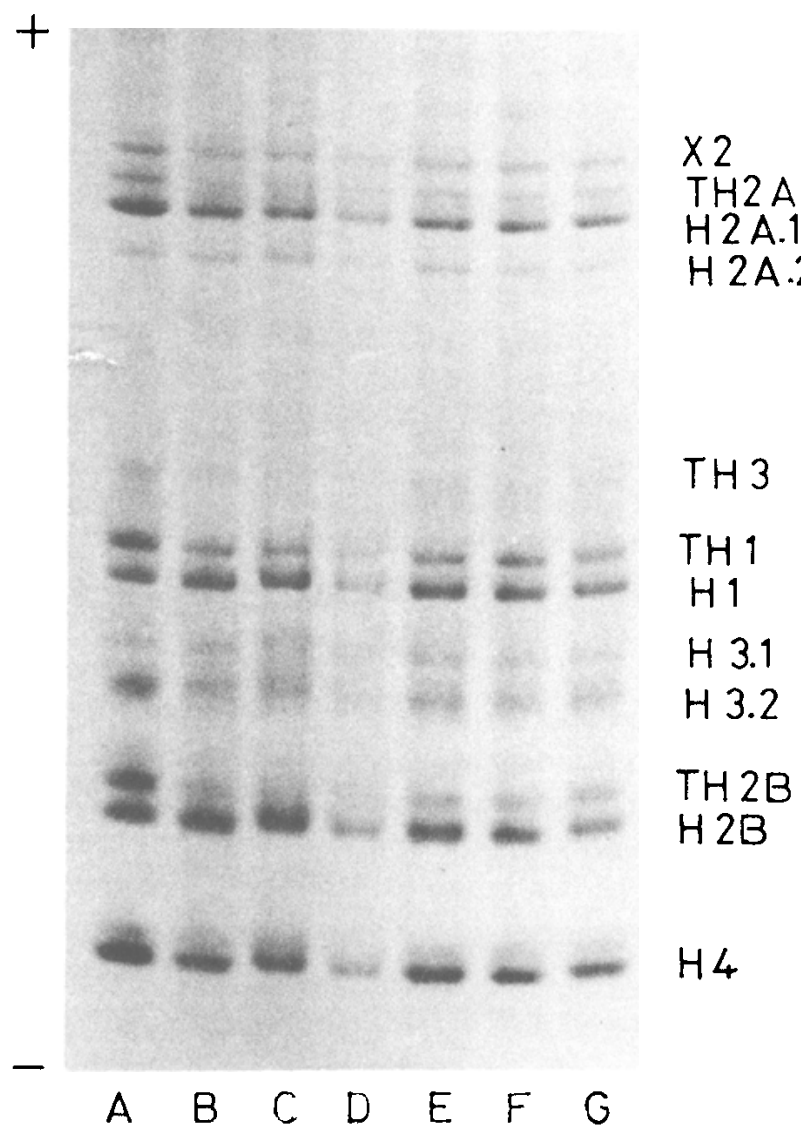

Figure 7. Electrophoretic analysis of acid-soluble nuclear proteins on a $12 \%$ Polyacrylamide Triton X-100-acid-urea gel. Approximately $100 \mu \mathrm{g}$ of total acid-soluble nuclear proteins from different rats were separated on a Triton-acid-urea-gel according to the method of Alfageme et al. (1974). A, Normal; B, retinoic acid maintained; C-G, retinyl acetate supplementation for C, 4 days; D, 8 days; E, 12 days; F, 16 days; and G, 24 days.

of this gel system was the separation of TH2B from H2B which was not possible in the acid-urea gel system. The position of TH2B in the acid-urea-Triton X-100 PAGE had been ascertained earlier (Rao et al., 1982). The electrophoretic separation of the acidsoluble nuclear proteins obtained from testes of differently treated rats are also shown in figure 7. A quantitative analysis of the peak areas of TH2A and TH2B obtained from the densitometric scans is presented in table 1 . It can be seen that the relative percentages of TH2A and TH2B out of total H2A's and H2B's respectively were significantly reduced in the testes of retinoate maintained rats (figrue $7 \mathrm{~B}$ and table 1 ). Again as observed above with histone Hlt, the levels of TH2A and TH2B further decreased when the retinoate maintained rats were supplemented with retinyl acetate for either 4 days or 8 days. However, their levels started increasing from 12 days of supplementation onwards, coinciding with the appearance of fresh wave of pachytene spermatocytes (figures 2E, 3 and table 1). 
Thus, the results presented here have shown that there is a good correlation between the levels of testis-specific histone variants, namely Hlt, TH2A and TH2B with the number and types of germ cells present in the seminiferous tubules of normal, vitamin A-depleted and vitamin A-repleted animals and have therefore shown that the testisspecific histone variants can be used to evaluate the status of spermatogenesis. In our earlier study, although we had observed the level of TP was greatly reduced in vitamin A-deficient rats, we did not detect any change in the levels of THl and other core histones (Rao et al., 1980). However, it should be clarified that those studies were done only on mildly vitamin A-deficient rats, while in the present study we have studied in greater detail the pattern of histones present in the testes of acutely deficient and also in retinoate maintained rats. Our studies have thus shown that one can use the levels of TP (Rao et al., 1980), Hlt, TH2A and TH2B as additional parameters to determine the stage at which spermatogenesis is arrested under any abnormal conditions, a proposal which has also been put forward by Chiu and Irvin (1978) based on their studies on cryptorchid testes.

The actual mechanism by which vitamin A supports the spermatogenic process is not clear at present. Recently, we have suggested that one of the primary sites of action of vitamin A is the Sertoli cells (Unni et al., 1983). Several recent studies have stressed the importance of Sertoli cells in maintaining normal spermatogenesis (Fritz, 1978; Russell, 1980). Since Sertoli cells do not divide in adult rats and their number do not change in vitamin A-deficient rats as shown in the present study, it is quite likely that they are probably defective in the deficient rats which can be corrected once vitamin $\mathrm{A}$ is supplemented. However, it remains to be seen whether the effect of vitamin A deficiency on germ cells is a direct one or mediated through Sertoli cells.

\section{Acknowledgements}

This work was financially supported by grants from the Department of Science and Technology, New Delhi. Emmanual Unni was under FIP programme of the University Grants Commission, New Delhi, and was also recepient of a Fellowship from the University of Kerala. We wish to thank Prof. G. Padmanaban for helpful discussions.

\section{References}

Alfageme, C. R., Zweidler, A., Mahowald, A. and Cohen, L. H. (1974) J. Biol. Chem., 249, 3729.

Brock, W. A., Trostle, P. K. and Meistrich, M. L. (1980) Proc. Natl. Acad. Sci. USA, 77, 371

Bucci, L. R., Brock, W. A. and Meistrich, M. L. (1982) Exp. Cell Res., 140, 111.

Chiu, M. L. and Irvin, J. L. (1978) Biol. Reprod., 19, 984.

Clermont, Y. (1972) Physiol. Rev., 52, 198.

Courot, M., Hochereall-de Riviers, M. T. and Ortavant, R. (1970) in The Testis (eds A. D. Johnson, W. R Gomes and N. L. Von Dermark) (New York: Academic Press) vol. 1, p. 339.

Fritz, I. B. (1978) in Biochemical Actions of Hormones (ed. G. Litwack) (New York: Academic Press) vol. 5, p. 249.

Gibson, D. R. and Gracy, R. W. (1979) Anal. Biochem., 96, 352.

Howell, J. M., Thompson, J. N. and Pitt, G. A. J. (1963) J. Reprod. Fertil., 5, 159.

Huang, H. F. S. and Hembree, W. C. (1979) Biol. Reprod., 21, 891.

Kumaroo, K. K., Jahnke, G. and Irvin, J. L. (1975) Arch. Biochem. Biophys., 168, 413. 
Laemmli, U. K. (1970) Nature (London), 227, 680.

Malathi, P., Subba Rao, K., Seshadri Sastry, P. and Ganguly, J. (1963) Biochem. J., 87, 305.

Mason, K. E. (1933) Am. J. Anat., 52, 153.

Meistrich, M. L, Brock, W. A., Grimes, S. R., Platz, R. D. and Hnilica, L. S. (1978) Fed. Proc., 37, 2522.

Mitranond, V., Sobhon, P., Tosukhowong, P. and Chidadungrat, W. (1979) Acta. Anat., 103, 159.

Panyim, S. and Chalkley, R. (1969) Arch, Biochem. Biophys., 130, 337.

Rao, M. R. S., Jagmohan Singh and Ganguly, J. (1980) Biochem. Biophys. Res. Commun., 94, 1.

Rao, M. R. S., Rao, B. J. and Ganguly, J. (1982) Biochem. J., 205, 15.

Russell, L. D. (1980) Gamete Res., 3, 189.

Seyedin, S. M. and Kistler, W. S. (1980) J. Biol. Chem., 255, 5949.

Trostle, P. K., Meistrich, M. L, Brock, W. A., Nishioka, F. and Bremer, J. W. (1982) J. Biol. Chem., 257,5560. Unni, E, Rao, M. R. S. and Ganguly, J. (1983) Indian J. Exp. Biol., 21, 891. 